EESTI NSV TEADUSTE AKADEEMIA TOIMETISED, 26. KÖIDE FUOSIKA * MATEMAATIKA. 1977, NR. 3

ИЗВЕСТИЯ АКАДЕМИИ НАУК ЭСТОНСКОИ ССР. ТОМ 26 ФИЗИКА * МАТЕМАТИКА. 1977, № 3

\title{
О РЕШЕНИИ ЗАДАЧ СУБОПТИМАЛЬНОЙ СТАБИЛИЗАЦИИ ДВИЖЕНИЙ НЕКОТОРЫХ ДИНАМИЧЕСКИХ СИСТЕМ МЕТОДОМ АГРЕГАЦИИ
}

Рассматриваются задачи субоптимального синтеза некоторых нелинейных динамических систем. Выражения субоптимальных регуляторов систем получены апрегацией $\left[{ }^{1,2}\right]$ системы порядка $n$ из агрегированной системы размерности $l \ll n$ и методом оптимальных функций Ляпунова [3,4]. Предлагаются различные варианты оптимизации по параметру агрегации с целью минимизации невязки и мажорантных функций. Результаты иллюстрируются на примере двух $y$-автономных $\left[{ }^{5}\right]$ механических систем, для которых получены решения задач оптимального управления по всем (некоторым) переменным.

1. Рассмотрим нелинейную динамическую систему

$$
\begin{gathered}
x^{*}=X(q)+A^{\prime}(q) u\left(q=\left(t, x_{\alpha}\right)^{*} \in R^{\prime}:|t|<\infty, \quad|x|<\infty\right), \\
x=\left(x_{\alpha}\right)^{*}, u=\left(u_{s}\right)^{*}, X(t, 0) \equiv 0, A^{\prime}=\left\|a_{\alpha s}(q)\right\|\left(X(q), A(q) \subset C_{1}\left(R^{\prime}\right)\right), \\
1 \leqslant \text { rank }\left\|a_{\sigma s}\right\|=r \leqslant n, u \in \Omega \subseteq E^{r}(x=d x / d t, \alpha=\overline{1, n ;} s, \sigma=\overline{1, r}),
\end{gathered}
$$

для которой решения (1.1) при любых $t \geqslant t_{0}, q_{0}=q\left(t_{0}\right) \in R^{\prime}$ существуют и единственны, если $u(t) \in\{u\}-$ множество кусочно непрерывных вектор-функций в $\Omega$. Условие оптимальности управления $u(t)-$ минимум интеграла

$$
I^{\prime}(u \mid q, a)=\int_{i}^{t_{1}} L^{\prime}(q, u) d \tau\left(L^{\prime}>0, y \neq 0, u \neq 0 ; L^{\prime} \subset C_{1}, q \in R^{\prime}, u \in \Omega\right),
$$

$t<t_{1}(a)=t_{1}$ - первый момент выполнения равенств $G_{j} \equiv y_{j}(q, a)=0$ $(j=1,2, \ldots, l \ll n)$. Переменные агрегации $y_{j}=y_{j}(q, a)$ содержат постоянный параметр $a=\left(a_{v}\right)^{*}(v=\overline{1, m} \leqslant \ln )$ из области $P \subseteq E^{m}(a=0 \in P)$, где выполнены условие гомеоморфности $x \leftrightarrow \xi$ $\xi=\left(y_{j}, x \beta\right)^{*}$ при $y \neq 0(\forall t=$ const, $\forall a \in P, \beta=\overline{l+1, n})$ и соотношения $y_{j}\left(t, 0, x_{2}, a\right) \equiv 0$,

$$
\begin{gathered}
\operatorname{det} \| \partial y_{j} / \partial x_{i}^{\prime}{ }_{i} \neq 0, \\
y_{j}(q, a) \subset C_{2}, y \neq 0\left(x_{1}=\left(x_{1}, \ldots, x_{l_{1}}\right)^{*}, x_{2}=\left(x_{l_{1}+1}, \ldots, x_{n}\right)\right)^{*},
\end{gathered}
$$

$y_{j}(q, a) \subset C\left(q \in R^{\prime}, a \in P\right), 1 \leqslant l_{1} \leqslant n, y \rightarrow 0$ при $x_{1} \rightarrow 0$ равномерно по $t, x_{2}, a$. Обозначим через $U^{\prime}\left(t, x_{2}, a\right)$ область значений $\left\{y_{j}\left(x_{1}\right)\right\}(i=\overline{1, l})$. Примем для простоты, что $\bigcap_{t, x_{2}, a} U^{\prime} \equiv U-$ неогра- 
ниченная область в $E^{l}(0 \in U)$. Последнее вместе с (1.3) выполняется в случае линейной $\left[{ }^{1,2}\right]$ агрегации $y=A^{0} x, \quad A^{0}=\left\|a_{j \alpha}^{0}\right\|=$ const, $\operatorname{rank} A^{0}=l, \operatorname{dim} a=m=l n$. На области $R^{0}:|t|<\infty, y \neq 0, y \in U$, $z \in E^{n-l}$ в переменных $\xi=\left(y_{j}, z_{\beta}\right)^{*}, z_{\beta} \equiv x_{\beta}$ система (1.1), (1.2) имеет при (1.3) вид

$$
\begin{gathered}
\xi=F_{0}(t, \xi, a)+A(t, \xi, a) u \equiv F(t, \xi, a, u)\left(F_{0}=\left(Y_{j}, Z_{\beta}\right)^{*}\right), \\
y=Y(t, \xi, a)+A_{1}(t, \xi, a) u, z=Z(t, \xi, a)+A_{2}(t, \xi, a) u, \\
Y=\left(Y_{j}\right)^{*}, \quad Y_{j}=X\left[y_{i}\right], X=\frac{\partial}{\partial t}+X_{\alpha} \frac{\partial}{\partial x_{\alpha}}, Z=\left(Z_{\beta}\right)^{*}, Z_{\beta}=\left(X_{\beta}(q)\right)^{*} \\
A=\left\|A_{A_{2}}^{A_{1}}\right\|, A_{1}=K A^{\prime}, K=\left\|k_{j \alpha}\right\|, k_{j \alpha}=\frac{\partial y_{j}}{\partial x_{\alpha}}, A_{2}=\left\|a_{\beta s}(q)\right\|, \\
I(u \mid t, \xi, a)=\int_{t}^{t_{1}} L(t, \xi, u, a) d \tau\left(L>0, \xi \neq 0, u \neq 0, L \subset C_{1}\left(R^{0} \times \Omega \times P\right)\right), \\
L(t, \xi, u, a) \equiv L^{\prime}(t, x(t, \xi, a), u), F_{0}(t, 0, a) \equiv 0 \quad\left(\xi \in R^{0}, u \in \Omega, a \in P\right) .
\end{gathered}
$$

Для $u(t) \in\{u\}$ в области $\Omega$ решения (1.4) при $t, t_{0}, \xi_{0} \equiv \xi\left(t_{0}\right) \in R^{0}$ существуют и единственны на $R^{0}$ в силу (1.1), (1.3). Задача оптимального по (1.2) приведения $x$ на $G_{j}=0$ сводится к $I$-оптимальному преобразованию $y$-компоненты решения $\xi(t)$. в нуль. Предположим, что $u(t) \in\{u\} \subset \Omega$, приводящим $y$ в нуль, отвечают в $R^{0} z$-продолжаемые решения (1.4). Для нахождения субоптимальных решений используем методы динамического пропраммирования Ляпунова $\left[{ }^{3,4}\right]$ и параметрической оптимизации по $a$. Пусть система (1.4) в $R \equiv R^{0} \cup y=0, \forall a \in P$ имеет непрерывную функцию $S(t, \xi, a)$, удовлетворяющую условиям

$$
S(t, 0, z, a) \equiv 0 \text {, }
$$

$S_{1}(y, a) \leqslant S(t, \xi, a) \leqslant S_{2}(y, a) \quad\left(S_{1,2}>0, y \neq 0, S_{1,2} \subset C\right.$ на $\left.R \times P\right)$,

$B[S, u] \geqslant B\left[S, u^{0}\right] \equiv 0, S \subset C_{1} ; u, u^{0} \in \Omega, u^{0}=u^{0}(t, \xi, \Theta, a) \subset C$ на $R^{0} \times P$, $\left.S \cdot\right|_{u=u_{0}} \equiv-L\left(t, \xi, u_{0}, a\right) \leqslant-w(y, a)$ на $R^{0}\left(S_{1}(0, a) \equiv S_{2}(0, a) \equiv 0\right.$ на $\left.P\right)$,

$$
\left(u_{0}=u_{0}(t, \xi, a)=u^{0}(t, \xi, \Theta(t, \xi, a), a)\right) .
$$

Здесь и ниже $f_{1,2}$, w- некоторые оценочные для $f y$-определенно положительные и непрерывные функции от $y, a$ и приняты обозначения

$$
B[f, u]=\frac{d f}{d t}+L(t, \xi, u, a)=\frac{\partial f}{\partial t}+y_{j} \frac{\partial f}{\partial y_{j}}+z_{v} \cdot \frac{\partial f}{\partial z_{\gamma}}+L \quad(y \neq 0),
$$

$\Theta=\left(p^{\prime}{ }_{j}, \psi_{\gamma}\right)^{*}, p^{\prime}{ }_{0}=\frac{\partial S}{\partial t}, p_{j}^{\prime}=\frac{\partial S}{\partial y_{j}}, \psi_{\gamma}=\frac{\partial S}{\partial z_{\gamma}}\left(z_{\gamma} \equiv z_{\beta}, j=\overline{1, l}, \gamma=\overline{1, n-l)}\right.$, $p^{1}=\left(p_{0}, p_{j}\right)^{*}, p=\left(p_{j}\right)^{*}, p_{0}=\frac{\partial V}{\partial t}, p_{j}=\frac{\partial V}{\partial y_{j}}\left(a_{i} b_{i} \equiv \sum_{i=1}^{N} a_{i} b_{i} \equiv a \cdot b, i=\overline{1, N}\right)$.

Допустим, что существует открытое множество $\{V(t, y, a)\}$ непрерывных функций $V$ на $R \times P ;\left|p^{1}\right|<\infty, V(t, y, a) \subset C_{1}$ на $R^{0} \times P$, все элементы которого имеют свойства

$$
V_{1}(y, a) \leqslant V(t, y, a) \leqslant
$$

$\leqslant V_{2}(y, a)\left(V_{1,2}(0, a) \equiv 0, V_{1,2}>0, y \neq 0, V_{1,2} \subset C\right.$ на $\left.R \times P\right)$, $0 \geqslant B\left[V, u^{1}\right], B\left[V, u^{1}\right] \leqslant B[V, u]\left(u, u^{1} \in \Omega, u^{1}=u^{1}(t, \xi, p, a) \subset C\right.$ на $\left.R^{0} \times P\right)$, 


\section{$L\left(t, \xi, u_{1}, a\right) \geqslant$}

$\geqslant w(y, a)\left(u_{1}(t, \xi, a) \equiv u^{1}(t, \xi, p(t, y, a), a) ; w>0, y \neq 0, w \subset C(R \times P)\right)$.

Если $R^{0} \times P-$ нормальная по $V$ область, то функция $u^{1}(t, \xi, p, a) \subset C$, $u^{1} \in \Omega$ и однозначна. Введем это условие в определение класса $\{V\}$.

Из (1.5)-(1.7) для $\forall a \in P$ и $\forall V \in\{V\}$ на основании результатов

$\left[{ }^{3-6}\right]$ находим, щто существует цилиндр $C(a):|y| \leqslant \delta, \delta=\delta(a)=$ const в $R^{0}$, где выполняются соотношения

$$
\begin{gathered}
I *+V(t, y, a) \leqslant S(t, \xi, a) \leqslant I^{*}+V(t, y, a) \equiv I_{1}^{1}\left(|V-S| \leqslant\left|I_{*}\right|\right), \\
S(t, \xi, a)=\min _{u \in \Omega} I(u \mid t, \xi, a), V(t, y, a)=\min _{u \in \Omega} I_{1}[V] \quad\left(u \in\{u\}, I_{1}[V] \equiv I_{1}\right), \\
I * \equiv \int_{t}^{t_{1}} b\left(\tau, \xi^{0}(\tau), a\right) d \tau, I^{*} \equiv \int_{t}^{t_{1}^{\prime}} b\left(\tau, \xi^{1}(\tau), a\right) d \tau \quad\left(I_{*} \leqslant I^{*} \leqslant 0\right), \\
I_{1}^{1} \equiv \int_{t}^{t_{1}^{\prime}} L\left(\tau, \xi^{1}, u_{1}, a\right) d \tau, I_{1} \equiv \int_{t}^{t_{1}^{\prime}} L(\tau, \xi, u, a) d \tau-\int_{t}^{t_{1}^{\prime}} b(\tau, \xi, a) d \tau, \\
b(\tau, \xi, a) \equiv B\left[V, u_{1}(t, \xi, a)\right] \leqslant 0, V_{1} \geqslant S_{1}, V_{2} \geqslant S_{2}, \\
S_{1}(y, a) \leqslant S(t, \xi, a) \leqslant V(t, y, a) \leqslant V_{2}(y, a) \quad(t, \xi \in C(a)),
\end{gathered}
$$

$t_{1}, t_{1}^{1}$ - первые моменты примыкания $0=y^{0}\left(t_{1}\right)=y^{1}\left(t_{1}^{1}\right)$ траекторий (1.4) $\xi^{0}(t), \xi^{1}(t)$, порожденных соответственно регуляторами $u_{0}, u_{1}$.

Неравенства (1.8) означают, что произведенный $V$-функцией вектор $u_{1}(t, \xi, a)$, оптимальный по $I_{1}=I_{1}[V]$ регулятор $y$-стабилизации, является субоптимальным регулятором системы (1.4) в $C(a)$. С учетом соотношений (1.3), аналогично $\left[{ }^{4,6}\right]$, получаем, что $u_{0}^{0}(q, a) \equiv$ $\equiv u_{0}(t, \xi(q, a), a)$ и $u_{1}^{0}(q, a) \equiv u_{1}(t, \xi(q, a), a)$ - соответственно оптимальный и субоптимальный по (1.2), минимизирующий $I_{1}{ }^{\prime} \equiv$ $\equiv I_{1}(u \mid t, \xi(q, a), a)$, регуляторы исходной задачи в цилиндре $C^{\prime}(a): y(q, a) \neq 0,\left|x_{1}\right| \leqslant \delta^{\prime}(a), \quad \delta^{\prime}=\delta^{1}(\delta, a)=$ const. При этом $\inf _{a \in P} I^{\prime}$ не зависит от $a$. $u_{1}, u_{1}^{0}$ - оптимальные по $I_{1}, I_{1}^{\prime}$ в $C(a), C^{\prime}(a)$ регуляторы $y$-стабилизации. Рассмотрим функционал

$I_{0}[V]=\int_{\omega_{0}} f\left(V, p^{1}, t, \xi, a\right) d \omega\left(f \subset C_{1}\right.$ на $\omega_{0} \times P$, где область $\left.\omega_{0} \subseteq R^{0}\right)$,

определенный и дифференцируемый на $\{V\}, \forall a \in P$, где $\|V\|=$ $=\sup |V|+\sup \left|p^{1}\right|$ на $\omega_{0} \subseteq R^{0}$. Для достаточно гладкой невязки $B$ положим $f=f_{0} \equiv B\left[V, u^{1}\right]$. Тогда из условия максимума (1.9) при $V^{*} \in\{V\}(B \leqslant 0)$

$$
\frac{\partial f}{\partial \Phi}-\frac{\partial}{\partial t}\left(\frac{\partial f}{\partial \Theta_{0}}\right)-\frac{\partial}{\partial \xi_{\alpha}}\left(\frac{\partial f}{\partial \Theta_{\alpha}}\right)=0 \quad\left(\Theta_{0} \equiv \frac{\partial \Phi}{\partial t}, \Theta_{\alpha} \equiv \frac{\partial \Phi}{\partial \xi_{\alpha}} \text { на } \omega_{0} X P\right)
$$

для определения $V^{*}$ получаем уравнение

$$
\frac{\partial}{\partial t}\left(\frac{\partial B^{1}}{\partial p_{0}}\right)-\frac{\partial}{\partial y_{j}}\left(\frac{\partial B^{1}}{\partial p_{j}}\right)=0 \quad\left(p_{0}=\frac{\partial V}{\partial t}, p=\frac{\partial V}{\partial y}\right)
$$

где $\quad B^{1}\left(t, \xi, p^{1}, a\right) \equiv B\left[V, u^{1}(t, \xi, p, a)\right] \quad$ на $\quad \omega_{0} \times P(\alpha=\overline{1, n ;} j=\overline{1, l})$. Если $\{V\} \ni V^{*}$ - единственное решение (1.10), то максимум (1.9) достигается лишь при $V \equiv V^{*}$ в случае $I_{0}\left[V^{*}\right] \geqslant I_{0}[V]$. Функцию $V^{*}$, максимизирующую (1.9), минимизируем на $P$ либо из условия близости 
$V^{*}(t, y(q, a), a)$ к $\inf I^{\prime}$ в точке const $=q_{0} \in C^{\prime}(a)$, либо в смысле некоторой меры близости на $Q$-области $\left(Q \subseteq R^{\prime}\right)$. Если класс $\{V\}-$ полный для (1.9) и нет максимизирующей $V^{*} \in\{V\}$, то максимизирующая последовательность $V_{n} \in\{V\}$ для $I_{0}$ строится по методу Ритца. Пусть система $[V(t, y, a)] \neq \varnothing$ и не образует класса $\{V\}$. Для ее элементов выбор параметра $a \in P$ подчиним либо условию максимума $I_{0}(a)$, либо условиям $a$-экстремальности величин, рассматриваемых в п. 2. Экстремальные значения $a^{0}$ вектора $a \in P$ для этих функций $f(a)$ будут $f$-оптимальными параметрами нелинейной агрегации (1.3), которые удовлетворяют уравнению $\partial f / \partial a^{0}=0$.

2. Рассмотрим вариант агрегации, где элиминация $z$ в (1.4) производится оператором вида max по $z$. Предполагается условие

$|z(t)| \leqslant n^{\prime}=$ const при $\left|z\left(t_{0}\right)\right| \leqslant n_{1} \leqslant n^{\prime}, y\left(t_{0}\right) \in U,\left|t_{0}\right|<\infty, u=u^{1}(t, \xi, p, a)$,

где $V=V^{0}(t, y, a), p=\partial V^{0} \partial y, u^{1}$ удовлетворяют неравенствам (1.7) в $N:|t|<\infty, y \in U,|z| \leqslant n^{\prime} \quad\left(N^{0}=N \backslash y=0\right)$. Этот вариант практически связан с системами $(1.1)$, у которых компонента $z=\left(x_{\beta}\right)^{*}$ ограничена в силу геометрических связей или устойчива при $\forall u \in\{x\}$ по Лагранжу, а также, когда $z(t)$ измерима и ограничена при неизвестной $z$.

Пусть для $\forall a \in P$ существуют $V^{0}(t, y, a)$ и $u^{1}(t, \xi, p, a)$, удовлетворяющие в $N^{0}$ условиям (1.7) и неравенству

$$
\max _{|z| \leqslant n^{\prime}} B\left[V^{0}, u^{1}\right] \equiv b^{*}(t, y, a) \leqslant 0 \text { на } N^{0}\left(u^{1}(t, \xi, p, a) \subset C\right) .
$$

Из (1.7), (2.1), (2.2), аналогично п. 1 , заключаем, что $\Omega \ni u_{1}(t, \xi, a) \equiv$ $\equiv u^{1}(t, \xi, p, a)$ - регулятор $y$-стабилизации системы (1.4), оптимальный по функционалу $I_{1}\left[V^{0}\right]$ в области $n^{0}(a)$ вида

$$
\begin{gathered}
n^{0}(a):\left|t_{0}\right|<\infty, 0<\left|y_{0}\right| \leqslant \delta^{0}(a)=\text { const, }\left|z_{0}\right| \leqslant n_{1}, \\
I_{1}\left[V^{0}\right]=\int_{t}^{t_{1}}\left(L(\tau, \xi, u, a)-B\left[V^{0}, u^{1}\right]\right) d \tau\left(V^{0}=\min _{\{u\} \in \Omega} I_{1}\left[V^{0}\right]\right),
\end{gathered}
$$

где $\delta^{0}>0$ находим $\left[{ }^{3,4}\right]$ по методу Ляпунова. Для субоптимального регулятора $u_{1}$ системы (1.4) получаем неравенство

$$
\inf _{\{u\} \in \Omega} I(u \mid t, \xi, a) \leqslant \int_{t}^{t_{1}} L\left(\tau, \xi, u_{1}, a\right) d \tau \equiv I_{1}^{0} \text { на } n^{0} \times P .
$$

В отличие от п. 1 решение оптимальной задачи в виде $S$ здесь не предполагается, единственность $u^{1}$ не требуется. Класс $\{V\}$ не вводится, условие $B\left[V^{0}, u^{1}\right] \leqslant 0$ следует из (2.2). При $a=0, b^{*} \equiv 0$ из (2.3), (2.4) для $y$-автономных систем $(1.1),(1.2)$ получаем известные $\left[{ }^{5}\right]$ результаты. Цилиндру $n^{0}(a)$ в переменных $t, x$ соответствует область $n_{1}^{0}(a)$ оптимальной управляемости $|t|<\infty, y(t, x, a) \neq 0 \quad\left|x_{1}\right| \leqslant \delta_{1}^{0}(a)=$ $=$ const, $\left|x_{\beta}\right| \leqslant n_{1} \quad(\beta=l+1, l+2, \ldots, n)$. Число $\delta_{1}^{0}=\delta_{1}^{0}\left(a, \delta^{0}\right)>0$ и вид $u_{1}^{0}(q, a) \equiv u_{1}(t, \xi(q, a), a)$ находим, обращая (1.3). Для известного решения $V=V^{0}(t, y, a), u^{1}\left[V^{0}\right]=u_{1}(t, \xi, a)$ задачи субоптимальной по системе (1.4) $y$-стабилизации подчиним выбор параметра агрегации в (1.3) условиям задач оптимизации по $a \in P$. Примем для простоты, что $\bigcap_{a \in P} n^{0}(a)$ содержит цилиндр $C^{0}:|t|<\infty, \quad 0<\left|y_{0}\right| \leqslant c^{0}=$ $=$ const, $|z| \leqslant n_{1}$. Заметим, что $\forall u_{1}(t, \xi, a)$ приводит любую $\xi_{0} \in C^{0}$ в нуль за бесконечное или конечное время $t_{1}\left(t_{0}, \xi_{0}, a\right)-t_{0}$. Оба случая трактуются совместно в практически достаточном варианте достижения 
поверхности $\Phi \equiv V\left(t, y, a_{*}\right)-\varepsilon=0 . \quad$ Здесь $\quad 0<$ const $=\varepsilon \leqslant \varepsilon_{1}$, $\sup _{|y| \leqslant \varepsilon_{1}} V_{2}\left(y, a_{*}\right) \ll \inf _{|y|=C^{0}} V_{1}\left(y, a_{*}\right), \varepsilon_{1}=\varepsilon_{1}\left(c^{0}\right)=$ const, $a_{*}-$ оптимальное

значение параметра следующей задачи.

Для точки $t, \xi \in C^{0}\left(V_{1}(y)>\varepsilon \rightarrow \Phi>0\right)$ требуется найти необходимые условия минимума по $a \in P$ функционала

$$
I_{1}^{*}(a)=\int_{t}^{t_{1}^{*}} L\left(t, \xi, u_{1}(t, \xi, a), a\right) d \tau\left(\Phi\left(t_{1}^{*}, y\left(t_{1}^{*}\right), a_{*}, \varepsilon\right)=0\right),
$$

где $\xi=\xi(\tau \mid a) \quad$ удовлетворяет (1.4) при $u=u_{1}(\tau, \xi \mid a)$. Здесь $t_{1}^{*}\left(t, \xi, a, a_{*}, \varepsilon\right)=t_{1}^{*}-$ первый момент выполнения связи (2.5). С учетом $(1.7),(2.4),(2.5)$ для разности $I_{1}^{0}(t, \xi, a), I_{1}^{*}(t, \xi, a)$ имеем оценку

$$
I_{1}^{0}-I_{1}^{*} \leqslant \varepsilon \quad\left(I_{1}^{0}=\int_{t}^{t_{1}} L\left(\tau, \xi, u_{1}, a\right) d \tau\right),
$$

которая означает непрерывность интеграла $I_{1}^{*}(\varepsilon)$ по $\varepsilon \geqslant 0$ в условии (2.5). Введем для импульсов и гамильтониана обозначения

$$
\lambda=\left(\mu_{j}, v_{\gamma}\right)^{*}, \quad G(t, \xi, a, \lambda)=\lambda \cdot F_{1}(t, \xi, a)+M(t, \xi, a),
$$

$F_{1} \equiv F\left(t, \xi, u_{1}(t, \xi, a), a\right), \quad M \equiv L\left(t, \xi, u_{1}(t, \xi, a), a\right) \quad(j=\overline{1, l} ; \gamma=\overline{1, n-l})$.

Оптимальное значение параметра $a$ * «минимизирует» гамильтониан на $\left[t, t_{1}^{*}\right]$

$$
\int_{t}^{t_{1}^{*}} \frac{\partial G}{\partial a_{v}^{*}} d \tau \equiv \int_{t}^{t_{1}^{*}}\left[\lambda_{*}(\tau) \frac{\partial F_{1}}{\partial a_{v}^{*}}+\frac{\partial M}{\partial a_{v}^{*}}\right] d \tau=0 \quad\left(\xi=\xi *=\xi\left(\tau \mid a_{*}\right), \overline{v=1, m}\right) .
$$

Здесь $\xi=\xi *(t), \lambda=\lambda_{*}(t)-$ начальное значение решения системы

$$
\xi_{*}=\partial G / \partial \lambda_{*}, \quad \lambda_{*}=-\partial G / \partial \xi_{*} \quad\left(\xi_{*}=\xi\left(\tau, a_{*}\right)\right)
$$

с $n+1$ граничными условиями

$$
\begin{gathered}
\mu_{j}\left(t_{1}^{*}\right)=v_{0} \partial V / \partial y_{j}\left(t_{1}^{*}\right), \quad v_{\gamma}\left(t_{1}^{*}\right)=0, \\
V\left(t_{1}^{*}, y\left(t_{1}^{*}\right), a_{*}\right)=\varepsilon \quad\left(t_{1}^{*}=T\left(t, \xi, a_{*}\right)=\mathrm{const}>t\right), \\
\mu_{j}(\tau)=\partial V / \partial y_{* j}(\tau), v_{\nu}(\tau)=0, \text { если } b\left(\tau, \xi, a_{*}\right) \equiv 0,
\end{gathered}
$$

где постоянные

$$
\begin{gathered}
0<\text { const }=v_{0}=M\left[t_{1}^{*}\right]\left(M\left[t_{1}^{*}\right]-b\left[t_{1}^{*}\right]\right)^{-1} \leqslant 1, \\
M\left[t_{1}^{*}\right]=M\left(t_{1}^{*}, \xi_{*}\left(t_{1}^{*}\right), a_{*}\right), \quad b\left[t_{1}^{*}\right]=b\left(t_{1}^{*}, \xi_{*}\left(t_{1}^{*}\right), a_{*}\right)
\end{gathered}
$$

имеют смысл (1.8), (2.6). Если соотношения (2.7)-(2.9) определяют единственное решение $a_{*}=a_{*}(t, \xi) \in P$ для данной точки $t$, $\xi \in C^{0}$, то абсолютный минимум (2.5) достигается лишь при $a=a_{*}$. Выбирая $\xi=\xi\left(q, a_{*}\right)$, получаем отсюда для (1.1) минимизирующее (1.2) значение параметра агрегации $a_{*}^{\prime}=a_{*}\left(t, \xi\left(q, a_{*}\right)\right)$ при $q \in n_{1}^{0}, \xi \in C^{0}$.

Выберем $q \in n_{1}^{0}$ так, чтобы $\xi \in C^{0}$ для любых $a \in P$. Рассмотрим мажорантную для $\inf _{u} I^{\prime}(u \mid q, a)$ функцию $V^{\prime} \equiv V^{\prime}(q, a) \equiv V^{0}(t, \xi(q, a), a)$. Для ее минимальности $\left(V^{\prime}\left(a^{0}\right) \leqslant V^{\prime}(a) a, a^{0} \in P\right)$ необходимо, чтобы 
$\partial V^{\prime} / \partial a^{0}=0$ в точке $q$. Так как $V^{0}(a) \geqslant I_{1}^{0}(a) \geqslant I_{1}^{*}(a), \quad$ то в общем случае имеем $a^{0} \neq a$. Следовательно, параметры, минимизирующие оценочную функцию $V^{0}$ и функционал $(2.5)$, вообще не совпадают. Ясно, что нахождение $a^{0}(t, x)$ из условий стационарности $V^{\prime}$ проще определения $a_{:^{\prime}}^{\prime}(t, x)$ из $(2.7)-(2.9)$.

Для выбора $V$-оптимального по мере параметра $a_{0}$ агрегации будем искать минимум функции

$$
f(a)=\int_{\omega_{1}} f_{0}\left(V^{\prime}(q, a), q\right) d \omega \quad\left(\omega_{1} \subseteq n_{1}^{1} \subseteq R^{\prime}, \xi\left(n_{1}^{1}\right) \subseteq C^{0}\right) .
$$

Здесь $f_{0}$ - достаточно гладкая, интегрируемая на области $\omega_{1}$ весовая функция от $V^{\prime}$ типа $\varrho(q) V^{\prime}(q, a)(\varrho(q) \geqslant 0)$.

Обсуждение. Изложенные результаты и постановку задач можно без особых изменений распространить на нелинейные по $u$ системы вида (1.1). Кроме того, достаточно использовать условие непрерывной дифференцируемости $V, S$ соответственно вдоль направлений $\left(1, F_{0 j}\left(t, \xi, u_{1}, a\right)\right)^{*}, \quad\left(1, F_{0 \alpha}\left(t, \xi, u_{0}, a\right)\right)^{*}(j=\overline{1, l} ; \alpha=\overline{1, n}) . \quad$ Преобразуя надлежащим образом выражения невязки в (1.9) и мажоранты в (2.2), получим аналогичные выводы для задач, в которых вместо $L>0$, $B\left[V, u_{1}\right] \leqslant 0, L\left(t, \xi, u_{1}, a\right) \geqslant W(y, a)$ достаточно требовать лишь $L \geqslant 0$, $0 \leqslant W(y, a) \leqslant L\left(t, \xi, u_{1}, a\right)-B\left(u_{1}\right)$. В качестве переменных агрегации можно предложить использовать параметрические связки от известных при $u \equiv 0$ функций Ляпунова, инвариантов и медленных переменных системы (1.1). В связи с этим возникает вопрос оптимального выбора функций $y_{j}(q, a)$ в соотношениях (1.3) относительно критериев типа минимума $\left|B\left[V, u_{1}\right]\right|$, либо из условий $y$-автономности переменных агрегации.

3. Пр и ме ры. В качеспве переменной агрегации $(l=1)$ рассмотрим скалярную функцию $y=\left|x_{1}\right|$ для $y$-автономных систем $[5,7,8]$

$$
\begin{gathered}
x_{1}^{*}=X_{1}(t, x)+u, \quad x_{2}^{*}=X_{2}(t, x)+A_{2}(t, x) u, \\
x_{1}=\left(x_{\alpha}\right)^{*}, \quad x_{2}=\left(x^{\prime}\right)^{*}, \quad x=\left(x_{\alpha}, x^{\prime}\right)^{*} \quad(\alpha=\overline{1, r ;} \quad \gamma=\overline{1, n-r)}, \\
X_{1}=\left(X_{\alpha}^{\prime}(t, x)\right)^{*}, \quad X_{2}=\left(X^{\prime}{ }_{\gamma}(t, x)\right)^{*}, \quad X_{\delta}(t, 0) \equiv 0, \quad A_{2}=\left\|a_{\gamma \alpha}(t, x)\right\|, \\
\operatorname{dim} x_{1}=\operatorname{dim} u=r, \quad|u| \leqslant M=\mathrm{const}, \quad 1 \leqslant r \leqslant n \quad(\delta=1,2),
\end{gathered}
$$

удовлетворяющих в области $t \geqslant 0,|x|<\infty$ соотношениям

$$
\begin{gathered}
X_{1} \cdot \partial y / \partial x_{1} \equiv Y_{1}(y), \quad y \neq 0 \quad\left(y \equiv\left|x_{1}\right| \geqslant 0\right), \\
y=Y_{1}(y)+y^{-1} x_{1} \cdot u, \quad y \neq 0 \quad\left(Y_{1}(y)<M, \quad Y_{1}(y) \subset C, y \geqslant 0\right) .
\end{gathered}
$$

Критерий оптимальности $x_{1}$-стабилизации - минимум затрат комбинации

$$
I_{2}^{1}=\int_{0}^{t_{1}}\left[f_{1}(y)|u|+f_{2}(y)\right] d \tau\left(M f_{1}+f_{2}>0, \quad Y_{1} f_{1}+-f_{2} \geqslant 0 \text { на } y \geqslant 0\right)
$$

типа времени-топлива. Из (1.5)-(1.8) с учетом (3.1), (3.2), $a=0$ получаем известные выражения оптимального регулятора и функции Ляпунова $[5,7,8]$

$$
u_{2}^{1}\left(x_{1}\right)=-M\left|x_{1}\right|^{-1} x_{1}, \quad \min _{|u| \leqslant M} I_{2}^{1}=V(y)=S_{1}=\int_{0}^{y}\left(M f_{1}-f_{2}\right)\left(M-Y_{1}\right)^{-1} d y .
$$

Подстановка (3.3) в (3.1) дает $y^{\circ}=Y_{1}(y)-M$. Отсюда следует, что 
$u_{2}^{1}\left(x_{1}\right)$ - оптимальный по (3.2) регулятор $x_{1}$-стабилизации в целом исходной механической системы.

Рассмотрим теперь ограниченную задачу стабилизации относительного равновесия гиростата $\left[{ }^{8-11}\right]$, образованного корпусом и роторами, оси которых закреплены вдоль его главных центральных осей. Предполагается, что вращение гиростата не влияет на равномерное движение его центра масс по окружности $r_{0}=$ const с угловой скоростью $\omega_{0}=$ $=\mathrm{const}=\mu^{1 / 2} \Gamma_{0}^{3 / 2}$ в нецентральном гравитационном поле массивного сфероида.

Уравнения возмущенного вращательного движения при стабилизации моментами $u_{1}, u_{2}$ от трех роторов и реактивных рулей, действующих через демпфирующий преобразователь с матрицей $R$, имеют в обозначениях [ $\left.{ }^{11}\right]$ вид

$$
\begin{gathered}
A \omega^{*}=\left(A \omega^{1}+z^{1}\right) \times \omega^{1}+3 \omega_{0}^{2}\left(\sigma^{1} \times G \sigma^{1}\right)+R_{1} u, \\
z=R_{2} u, \quad \sigma=\sigma^{1} \times\left(\omega^{1}-\omega_{0} \gamma^{1}\right), \quad \gamma=\gamma^{1} \times \omega^{1}, \\
\omega=\omega^{1}-\omega^{0}, \quad z=z^{1}-z^{0}, \quad \sigma=\sigma^{1}-\sigma^{0}, \quad \gamma=\gamma^{1}-\gamma^{0},
\end{gathered}
$$

$\omega^{0}=\left(0,0, \omega_{0}\right)^{*}, \quad z^{0}=\left(0,0, z_{0}\right)^{*}, \quad \sigma^{0}=(1,0,0)^{*}, \quad \gamma=(0,0,1)^{*} \quad\left(z_{0}=\right.$ const $)$, $A=\operatorname{diag}\left(A_{1}, A_{2}, A_{3}\right)>0, \quad G=\operatorname{diag}\left(G_{1}, G_{2}, G_{3}\right)>0, \quad G-A>0$, $u=\left(u_{s}\right)^{*}=\left(u^{\prime \prime}{ }_{k}-u_{k}^{\prime}, u_{k}^{\prime}\right)^{*}, u_{1}=\left(u_{k}^{\prime}\right)^{*}, u_{2}=\left(u^{\prime \prime}\right)^{*}, \quad(s=\overline{1,6} ; k=\overline{1,3})$, $R=|v| R^{0}=\left\|\begin{array}{l}R_{1} \\ R_{2}\end{array}\right\|, \quad R^{0}=\left\|r_{s \sigma}^{0}\right\|=$ const, $\operatorname{rank} R^{0}=6 \quad(\sigma, s=\overrightarrow{1,6})$, $v^{*}=\left\|A_{1}\left(\omega_{1}-\beta_{1}\right), A_{2}\left(\omega_{2}-\beta_{2}\right), A_{3}\left(\omega_{3}-\beta_{3}\right), c_{1} z_{1}-\beta_{1}, c_{2} z_{2}-\beta_{2}, c_{3} z_{3}-\beta_{3}\right\|$, $\beta=\omega_{0} \gamma, c_{k}=$ const $>0, \operatorname{dim} \sigma, \gamma, \omega, z=3\left(v=l\left(\gamma, \omega, z \mid c_{k}\right)\right)$,

$\omega^{1}, z^{1}, \sigma^{1}, \gamma^{1}$ обозначают вектор-функции возмущенного движения.

Задачей оптимальной стабилизации движения $\omega=0, z=0, \sigma=0$, $\gamma=0$ является гашение компоненты $x_{1}=\left(x_{i}\right)^{*}=\left(\sigma_{\alpha}, \gamma_{k}, \omega_{k}, z_{k}\right)^{*}(\alpha=2,3)$ возмущения $x=\left(x_{j}\right)^{*}=\left(\sigma_{1}, x_{i}\right)^{*}(j=\overline{1,12} ; i=\overline{2,12})$ при условии минимума интеграла

$$
\begin{gathered}
I_{2}=\int_{0}^{t_{1}} \lambda(v) f(w, y) d \tau \quad\left(\lambda \equiv|v|\left|R^{0} v\right| \geqslant 0\right), \\
w \equiv|u| \leqslant M=\mathrm{const}, \quad f(M, y)>0, \quad M f(w, y) \geqslant w f(M, y), \\
f(w, y) \subset C \quad \text { при } 0 \leqslant w \leqslant M, \quad 0 \leqslant y .
\end{gathered}
$$

Здесь $y=y\left(x_{1}, c_{0}\right)$ - положительно определенный по $x_{1}$ инвариант системы (3.4) при $u \equiv 0$, содержащий положительный скалярный параметр $c_{0}=$ const

$$
\begin{gathered}
2 y=\underset{\omega_{0}^{2}}{2} B_{\alpha} \sigma_{\alpha}^{2}+\stackrel{2}{\omega_{0}} A_{0} \gamma \cdot \gamma-2 \omega_{0} A \omega \cdot \gamma+A_{k} \omega_{k}^{2}-2 \omega_{0} \gamma \cdot z+c_{k} z_{k}^{2}, \\
B_{\alpha} \equiv 3\left(G_{\alpha}-G_{1}\right), A_{0} \equiv A_{3}+z_{0} \omega_{0}^{-1}, c_{k}=\left(c_{0}-3 G_{k}-A_{k}\right)^{-1}>0
\end{gathered}
$$

при условиях, что $A_{k}, G_{k}, z_{0}, c_{0}$ удовлетворяет неравенствам

$$
\max A_{k}<A_{3}+z_{0} \omega_{0}^{-1}, G_{\alpha}-G_{1}>0 \quad(\alpha=\overrightarrow{2,3} ; k=\overrightarrow{1,3)},
$$

$\max A_{k}+3 \max G_{\alpha}<c_{0}<A_{0}+3 G_{1}+\min A_{k}-\max A_{k}$ (const). 
Аналогично рассмотренному примеру получаем выражения граничного регулятора и оптимальной функции

$$
\begin{gathered}
u_{2}^{0}=u_{2}^{0}\left(\omega, z, \gamma, c_{0}\right)=-M\left|R^{0} v\right|^{-1} R^{0^{*} v}, \\
V=V(y)=S_{2}\left(x_{1}, c_{0}\right)=\int_{0}^{y} M^{-1} f(M, \xi) d \xi .
\end{gathered}
$$

Переменная агрегации $y=y\left(x_{1}, c_{0}\right)$ в силу (3.4), (3.8) удовлетворяет уравнению $y^{*}=-M \lambda(v)=-M\left|v \| R^{0} v\right| \leqslant 0$. Используя (3.6) и геометрические инварианты системы (3.4)

$$
2 \sigma_{1}+\sigma \cdot \sigma=0, \sigma_{3}+\gamma_{1}+\sigma \cdot \gamma=0,2 \gamma_{3}+\gamma \cdot \gamma=0,
$$

находим, что инвариантное множество (3.4) при $u=u_{2}{ }^{0}$ содержит в плоскости $v=0$ лишь восемь перманентных вращений гиростата вокруг бинормали $\gamma=\sigma \times v$ к плоскости его орбиты $\left(r^{*}=r_{0} \omega_{0} v\right)$

$$
x_{1}^{1}=(0, \ldots, 0)^{*}, x_{2}^{1}=(-2,0, \ldots, 0)\left(\operatorname{dim} x_{p}^{1}=12, p=\overline{1,8}\right) \text {, }
$$

$x_{3}^{1}=(-1,+1,0, \ldots, 0)^{*}, x_{4}^{1}=(-1,-1,0, \ldots, 0)^{*}, x_{5}^{1}=\left(0,0, \delta_{q}\right)^{*}$,

$x_{6}^{1}=\left(-2,0, \delta_{q}\right)^{*}, x_{7}^{1}=\left(-1,+1, \delta_{q}\right)^{*}, x_{8}^{1}=\left(-1,-1, \delta_{q}\right)^{*}$,

$$
\delta_{4}=-2, \delta_{7}=-2 \omega_{0}, \delta_{10}=-2 \omega_{0} c_{3}^{-1}, \delta_{q}=0, q \neq 4,7,10(q=\overline{1,10}) .
$$

Траектории системы (3.4) при $u=u_{2}{ }^{0}$ примыкают к (3.9) за бесконечный промежуток времени.

Рассмотрим уравнение $y^{\cdot}=-M \lambda(v)$ в области $y\left(x_{1}, c_{0}\right)<h^{0} \equiv$ $\equiv 2 \inf y\left(\left|x_{1}\right|^{-1} x_{1}\right)$, где нет точек (3.9), отличных от $x_{1}{ }^{1}=0$. Из теоремы Барбашина-Красовского следует, что в этой области регулятор (3.8) реализует $x_{1}$-стабилизацию относительного равновесия гиростата, оптимальную по (3.5). При этом из первого геометрического инварианта находим либо неравенство $\left|\sigma_{1}(t)+2\right|<\varepsilon$ при $t \geqslant T_{1}(\varepsilon)$, либо неравенство $\left|\sigma_{1}(t)\right|<\varepsilon$ при $t \geqslant T_{2}(\varepsilon)$, которые означают соответственно «зеркальную» и полную стабилизацию $\left(t_{1}=f \infty\right)$. В этом примере $V=$ $=V(y)$ не зависит от $c_{0}$ явно. Выбор параметра $c_{0}=$ const можно подчинить условию минимизации $\operatorname{tr} Q\left(Q=\right.$ const, $\left.\dot{x}_{1}^{*} Q x_{1}=2 y\right)$ с целью увеличения области управляемости либо минимизации $y^{*}$ по $c_{0}$ с учетом последнего из условий (3.7).

Существенно отметить, что при отсутствии демпфирования ( $R=$ const) применение регулятора $u_{2}^{0}$ требует рассмотрения скользящих режимов на множестве $v=0$. При отключенных газовых рулях $\left(u_{2} \equiv 0\right)$ результаты примера можно модифицировать для случая управления с одним маховиком $\quad\left(u_{1}=w^{\prime}\left(a_{1}^{0}, a_{2}^{0}, a_{3}^{0}\right) *, a_{k}^{0}=\right.$ const, $k=$ $=\overline{1,3)}$. В примерах используется расширенная система - объединение исходной с уравнением эволюции переменной агрегации. Сохраняя основные из рассмотренных рассуждений, этот прием нетрудно распространить на общий случай, чтобы уменьшить затруднения, связанные с получением значений $x=x(t, \xi, a)$. При этом равенства $y_{j}-$ $-y_{j}(t, x, a)=0$ определяют ограничения на переменные расширенной системы. 


\section{Л И Т Е Р А Т У Р А}

1. W ismer, D. A., A oki, M., Optimization methods for large-scale systems, New York, 1971, p. 191-232.

2. У л ь м С., Изв. АН ӘССР, Физ. Матем., 20, 3 (1971).

3. К р асовски й Н. Н., В кн.: Малкин И. Г., Теория устойчивости движений, изд. 4, М., 1966.

4. Р у м нцев В. В., ПММ, 34, вып. 3, 440 (1970).

5. К е й с И., Изв. АН ЭССР, Физ. Матем., 26, 37 (1977).

6. К е й с И., Изв. АН ЭССР, Физ. Матем., 24, 178 (1975).

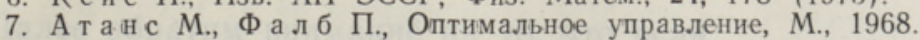

8. Л то в А. М., Динамика полета и управления, М., 1969.

9. Р у мянце в В. В., Об устойчивости стационарных движений спутников, М., 1967.

10. К ре ментуло В. В., ПММ, 34, вып. 5, 965 (1970).

11. К е й с И., Изв. АН ЭССР, Физ. Матем., 24, 277 (1975).

Ннститут кибернетики

Академии наук Эстонской ССР
Поступила в редакцию 18/VI 1976

\section{KEIS}

\section{LIIKUMISE SUBOPTIMAALSE STABILISEERIMISE OLESANNETE LAHENDAMISEST AGREGEERIMISMEETODIL MÕNEDE DUNAAMILISTE SUSTEEMIDE KORRAL}

Vaadeldakse suboptimaalset sünteesi mõnedes mittelineaarsetes dünaamilistes süstcemides. Suboptimaalsed regulaatorid saadakse optimaalsete funktsioonide meetodit ja agregeerimist kasutades. Esitatakse vea ja majorantfunktsioonide parameetrilise minimeerimise meetodid. Kahe $y$-autonoomse süsteemi korral annavad tulemused $y$-optimaalse stabiliseerimise ülesannete lahendi.

\section{KEIS}

\section{ON SUBOPTIMAL STABILIZATION PROBLEM SOLUTION FOR A DYNAMICAL SYSTEM VIA AGGREGATION METHOD}

In the paper the suboptimal stabilization problem for a nonlinear system is considered. The suboptimal control is found on the basis of Lyapunov's direct method and dimension reduction in aggregation. Optimal aggregation parameter choice is submitted to various minimum conditions. Exact optimal solutions are obtained for two $y$-autonomous mechanical systems as examples. 